\title{
Substrate polarization effects in two-dimensional magnetic arrays
}

\author{
N. Mikuszeit, ${ }^{1, *}$ F. Luo, ${ }^{1}$ G. Gómez-Santos, ${ }^{2}$ J. Camarero, ${ }^{1,2}$ and R. Miranda ${ }^{1,2}$ \\ ${ }^{1}$ Instituto Madrileño de Estudios Avanzados en Nanociencia, IMDEA-Nanociencia, Campus Universidad Autónoma \\ de Madrid, 28049 Madrid, Spain \\ ${ }^{2}$ Departamento de Física de la Materia Condensada and Instituto Nicolas Cabrera, Universidad Autónoma de Madrid, \\ 28049 Madrid, Spain
}

(Received 24 April 2012; revised manuscript received 5 July 2012; published 15 August 2012)

\begin{abstract}
The magnetostatic energy of a two-dimensional (2D) periodic array of magnetic particles (or a thin film with periodic magnetization) is evaluated, including additional energy terms due to a polarizable substrate. The polarization of the substrate is solved self-consistently using surface charges. This requires describing the magnetic potential of the 2D array in terms of an equivalent surface charge distribution. Analytic expressions for the magnetostatic self-energy of the $2 \mathrm{D}$ array as well as the energy due to the interaction of the magnetic structure and polarizable substrate are presented. It is shown how substrates with large susceptibility significantly alter the stray-field energy and, hence, the magnetic properties of the array, even promoting a spin-reorientation transition. Our results suggest that system properties can be controlled in a simple way by exploiting substrates with tunable polarizability.
\end{abstract}

DOI: 10.1103/PhysRevB.86.054423

PACS number(s): 75.75.-c, 41.20.Gz, 02.30.Em

\section{INTRODUCTION}

Nowadays magnetism deals with more and more miniaturized systems down to single magnetic atoms on surfaces. ${ }^{1}$ Nevertheless, many properties such as hysteresis are still described in a quasiclassical manner. ${ }^{2}$ This is even more the case if one has to consider the stray-field properties of systems used in technical applications. ${ }^{3-5}$ In the case of particles and thin films classical potential theory and continuum theory describe the stray field sufficiently, even if the characteristic dimensions are only of a few angstroms. On the one hand, the stray or demagnetizing field of a single particle becomes less important with decreasing particle size as the total magnetic volume decreases. On the other hand, due to the long-range character of the dipole-dipole interaction stray-field energies are still important if one has to consider large arrays of interacting small particles. 6 Hence, on the nanoscale-especially with respect to the superparamagnetic limit — a detailed knowledge of the stray field and stray-field energies remains important.

The stray-field problem consists in the solution of the Poisson equation such that results strongly depend on the magnetization distribution and on boundary conditions. ${ }^{7}$ Although the boundary value problem has a general solution, ${ }^{8,9}$ several symmetry properties may reduce the general complexity. Among other structures with simplified solutions are homogeneously magnetized cuboids, ${ }^{10}$ two-dimensional periodic arrays of homogeneously magnetized rectangular elements, ${ }^{11}$ or thin films with periodic quasi-one-dimensional ${ }^{12}$ and twodimensional ${ }^{13}$ magnetization structures.

In this work we show that a strongly polarizable substrate can significantly influence the energetics of magnetic arrays. The effects are similar to a soft magnetic underlayer (SUL) introduced below a hard magnetic material as in magnetic recording devices. ${ }^{14,15}$ We present simplified analytical solutions for two-dimensional (2D) arrays or thin films with a $2 \mathrm{D}$ periodic magnetization distribution that is constant in $z$. The solutions naturally include antidot arrays, such that the presented results are also interesting for magnonic applications. ${ }^{16}$ The results further take into account a semiinfinite polarizable substrate, an unavoidable contribution in every experiment. It is shown that the substrate stabilizes perpendicular magnetization in patterned media. Note that in the case of magnonics, i.e., in the $\mathrm{GHz}$ regime, one should carefully consider the substrate's time constants. Finally, all effects described here also hold for ferroelectric materials on top of an electrically polarizable substrate. ${ }^{17}$

\section{EQUIVALENT SURFACE CHARGES}

To calculate the magnetostatic energy, let us assume a quasitwo-dimensional magnetization distribution that is periodic in the $x-y$ plane and within the limits $-h<2 z<h$. A special case of such a distribution is shown in Fig. 1, where the 2D periodicity is due to the geometrical structuring into cuboids while the magnetization inside the cuboids is constant. The magnetization can be written in the form $\left(M_{x}, M_{y}, M_{z}\right)=$ $M_{\mathrm{S}}\left(a_{x}(\vec{\rho}), a_{y}(\vec{\rho}), b(\vec{\rho})\right),{ }^{18}$ where $\rho=(x, y)$. The functions $a_{i}$ and $b$ naturally fulfill $a_{x}^{2}(\vec{\rho})+a_{y}^{2}(\vec{\rho})+b^{2}(\vec{\rho})=1$ and have the Fourier coefficients $a_{q, x}, a_{q, y}$, and $b_{q}$. Defining $\vec{a}_{q}=$ $\left(a_{q, x}, a_{q, y}\right)$ the magnetic potential $\Phi$ of such a distribution has the form

$$
\Phi(\vec{r})=-\frac{M_{\mathrm{S}}}{2} \sum_{\vec{q}} \mathrm{e}^{2 \pi \mathrm{i} \vec{q} \cdot \vec{\rho}}\left[\mathrm{i} \frac{\vec{a}_{q} \cdot \vec{q}}{|\vec{q}|} t(|\vec{q}|, h, z)-b_{q} u(|\vec{q}|, h, z)\right],
$$

where $\vec{q}=\left(k / \lambda_{x}, j / \lambda_{y}\right)-(k, j) \in \mathbb{Z}^{2}$-is the wave vector divided by $2 \pi$ and $^{12}$

$$
t(q, h, z)= \begin{cases}\frac{\sinh (\pi q h)}{\pi q} \mathrm{e}^{-2 \pi q|z|} ; & |z|>\frac{h}{2}, \\ \frac{2-\exp (-\pi q|h+2 z|)-\exp (-\pi q|h-2 z|)}{2 \pi q} ; & |z| \leqslant \frac{h}{2}\end{cases}
$$

and

$$
u(q, h, z)= \begin{cases}\operatorname{sgn}(z) \frac{\sinh (\pi q h)}{\pi q} \mathrm{e}^{-2 \pi q|z|} ; & |z|>\frac{h}{2}, \\ \frac{\sinh (2 \pi q|z|)}{\pi q} \mathrm{e}^{-\pi q h} ; & |z| \leqslant \frac{h}{2} .\end{cases}
$$




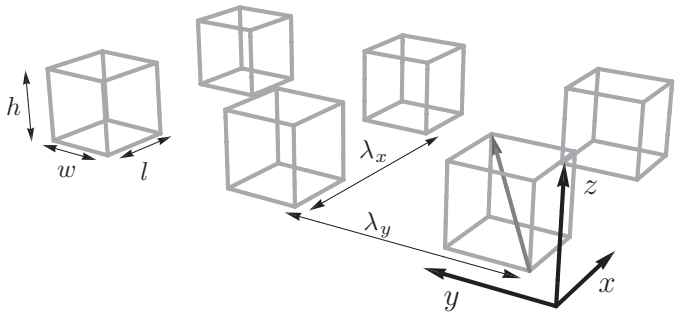

FIG. 1. Square lattice of magnetic cuboids of dimensions $l \times w \times$ $h$. The array with lattice constants $\lambda_{x}$ and $\lambda_{y}$ is on top of a substrate with the interface at $2 z=-h$. A magnetization in the [111] direction is sketched as dark gray arrow.

In contrast to the rather complicated expression for the potential of a magnetization distribution, the potential $\Phi_{\mathrm{C}}$ of a periodic $2 \mathrm{D}$ charge distribution at $2 z=-h$ reads

$$
\Phi_{\mathrm{C}}(\vec{r})=\frac{\sigma_{0}}{4 \pi \mu_{0}} \sum_{\vec{q}} \mathrm{e}^{2 \pi \mathrm{i} \vec{q} \cdot \vec{\rho}} s_{q} \frac{\mathrm{e}^{-\pi|\vec{q}||2 z+h|}}{|\vec{q}|} .
$$

Note that the charge distributions is truly $2 \mathrm{D}$; i.e., $\sigma(x, y, z)=$ $\sigma(\vec{\rho}) \delta(2 z+h)$. The total charge of a unit cell sums up to zero, i.e., $s_{q}=0$ for $q=0$, as the charge has its source in the magnetic structure. The factors $s_{q}$ are the Fourier coefficients of the function $s(\vec{\rho})$, where $\sigma(\vec{\rho})=\sigma_{0} s(\vec{\rho})$, and it is convenient to set $\sigma_{0}=\mu_{0} M_{\mathrm{S}}$.

For $2 z<h$ as well as for $2 z>h$ the potential $\Phi$ of the periodic magnetic structure can be given in the form of an equivalent surface charge, which is placed at $2 z=-h$ or $2 z=$ $h$, respectively. In each of the two cases the equivalent surface charge does not describe the potential in the other half space as this space contains sources of the field, namely the magnetic $2 \mathrm{D}$ structure. If the magnetic array is supposed to be on top of a polarizable substrate, the substrate is affected by the potential for $2 z<-h$, which can be described by a surface charge at $2 z=-h$.
Comparing Eq. (1) and Eq. (4) the Fourier coefficients of the equivalent surface charge have the form

$$
s_{q}=\left(\mathrm{e}^{-2 \pi|\vec{q}| h}-1\right)\left(\mathrm{i} \frac{\vec{a}_{q} \cdot \vec{q}}{|\vec{q}|}+b_{q}\right) .
$$

The potential of a magnetization distribution and the potential of its corresponding equivalent surface charge are shown in Fig. 2.

\section{SELF-CONSISTENT SOLUTION FOR THE SUBSTRATE POLARIZATION}

In general the substrate that supports the 2D magnetic array is polarized due to the stray field of the array. Moreover, the polarization produces a stray field modifying the total energy, i.e., the magnetostatic energy of the array as well as of the substrate itself. If the polarization of the substrate is proportional to the local field, i.e., the tensor of polarizability is isotropic, it can be substituted by a scalar. In this case the polarization of the substrate only produces surface charge. This is due to the fact that the field inside the substrate has zero divergence-there are no sources. To stress the point, for isotropic polarizability a polarization due to the field of a surface charge distribution only creates a surface charge, i.e., modifies the initial distribution. From a mathematical point of view, this is a huge difference to a soft magnetic layer. Hence, if the magnetic array produces a surface charge distribution $\sigma_{0}^{\text {stat }}$, the polarization results in $\sigma_{0}^{\text {ind }}$, where the polarization naturally is caused by $\sigma_{0}^{\text {stat }}+\sigma_{0}^{\text {ind }}$.

As the polarization only has surface charge the selfconsistent calculation of $\sigma_{0}^{\text {ind }}$ only has to consider the $z$ component of the field at the surface. The self-consistent potential $\Phi_{\mathrm{sc}}$ reads

$$
\Phi_{\mathrm{sc}}(\vec{r})=\frac{M_{\mathrm{S}}}{4 \pi} \sum_{\vec{q}} \mathrm{e}^{2 \pi \mathrm{i} \vec{q} \cdot \vec{\rho}}\left(s_{q}^{\text {stat }}+s_{q}^{\text {ind }}\right) \frac{\mathrm{e}^{-\pi|\vec{q}||2 z+h|}}{|\vec{q}|},
$$
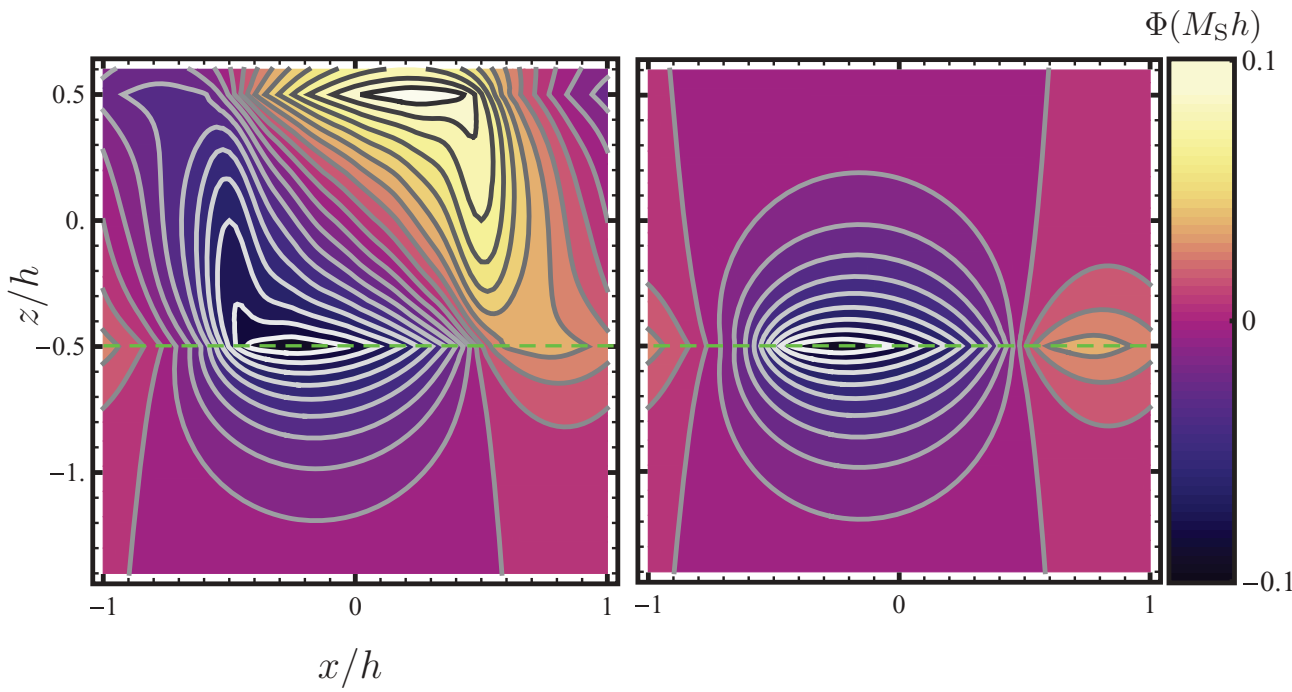

FIG. 2. (Color online) Magnetic potential of an array of cubes $\left(l=w=h\right.$ and $\lambda_{x}=\lambda_{y}=2 h$ ) magnetized in the [111] direction. Contour plot of the $x-z$ plane for $y=0$ (left). The potential due to the equivalent surface charge is shown in the right panel. The two solutions are identical for $2 z \leqslant-h$, but differ for $2 z>-h$, as the magnetic array has sources of the field inside this volume. Hence, the divergence of the potential is nonzero here, while an equivalent surface charge can only reproduce the potential in a region of zero divergence. 
where $s_{q}^{\text {ind }}$ are the Fourier coefficients of the induced charge normalized by $\sigma_{0}$. The $z$ component of the field at the surface is

$$
\begin{aligned}
\left.H_{z}\right|_{\text {surface }}= & -\left.\frac{\mathrm{d}}{\mathrm{d} z} \Phi_{\mathrm{sc}}(\vec{r})\right|_{\text {surface }}=\lim _{z \nearrow-\frac{h}{2}} \frac{M_{\mathrm{S}}}{2} \sum_{\vec{q}} \mathrm{e}^{2 \pi \mathrm{i} \vec{q} \cdot \vec{\rho}} \\
& \times\left(s_{q}^{\text {stat }}+s_{q}^{\text {ind }}\right) \frac{\operatorname{sgn}(2 z+h)}{\mathrm{e}^{\pi|\vec{q}||2 z+h|}} \\
= & -\frac{M_{\mathrm{S}}}{2} \sum_{\vec{q}} \mathrm{e}^{2 \pi \mathrm{i} \vec{q} \cdot \vec{\rho}}\left(s_{q}^{\text {stat }}+s_{q}^{\text {ind }}\right) .
\end{aligned}
$$

The induced charge distribution can be expressed either as

$$
\sigma^{\text {ind }}=\mu_{0} \chi \lim _{z \nearrow-\frac{h}{2}} H_{z}(z)=-\chi \frac{\sigma_{0}}{2} \sum_{\vec{q}} \mathrm{e}^{2 \pi \mathrm{i} \vec{q} \cdot \vec{\rho}}\left(s_{q}^{\text {stat }}+s_{q}^{\text {ind }}\right)
$$

or as

$$
\sigma^{\text {ind }}=\sigma_{0} \sum_{\vec{q}} \mathrm{e}^{2 \pi \mathrm{i} \vec{q} \cdot \vec{\rho}} s_{q}^{\text {ind }} .
$$

Comparing Eqs. (8) and (9) one easily gets

$$
s_{q}^{\text {ind }}=-\frac{\chi}{2+\chi} s_{q}^{\text {stat }}
$$

Note that as the substrate provides a surface charge with similar Fourier coefficients, only scaled by $-\chi(2+\chi)^{-1}$, it can be interpreted as the field of a scaled mirror image of the magnetic structure. This interpretation gives an intuitive picture of the interaction. The self-consistent solution for the potential inside the substrate then reads

$$
\Phi_{\mathrm{sc}}(\vec{r})=\frac{2}{2+\chi} \frac{M_{\mathrm{S}}}{4 \pi} \sum_{\vec{q}} \mathrm{e}^{2 \pi \mathrm{i} \vec{q} \cdot \vec{\rho}} s_{q}^{\text {stat }} \frac{\mathrm{e}^{-\pi|\vec{q}||2 z+h|}}{|\vec{q}|} ;
$$

i.e., the potential inside the substrate is reduced by the factor of $2(2+\chi)^{-1}$.

\section{MAGNETOSTATIC ENERGIES}

The knowledge of $s_{q}^{\text {ind }}$ enables the calculation of the total magnetostatic energy of the magnetic-structure-substrate system, which can be decomposed into two parts, the selfenergy of the periodic magnetic structure, and the energy gained by bringing the structure into contact with the substrate.

The self-energy volume density of the magnetic structure has the form

$$
\begin{aligned}
\frac{\mathcal{E}^{\text {stat }}}{K_{\mathrm{d}}}= & \sum_{\vec{q}}\left[\frac{\left|\vec{q} \cdot \vec{a}_{q}\right|^{2}}{|\vec{q}|^{2}}\left(1-\frac{1-\mathrm{e}^{-2 \pi|\vec{q}| h}}{2 \pi|\vec{q}| h}\right)\right. \\
& \left.+\left|b_{q}\right|^{2} \frac{1-\mathrm{e}^{-2 \pi|\vec{q}| h}}{2 \pi|\vec{q}| h}\right],
\end{aligned}
$$

where $2 K_{\mathrm{d}}=\mu_{0} M_{\mathrm{S}}^{2}$. This form is a generalization of the formulas given in Refs. 11 and 12. A more general but rather complicated form, not restricted to a confinement in $-h<2 z<h$, is given in Ref. 19. Note that the energy is given per volume and not per magnetic volume. To get the latter one, naturally Eq. (12) has to be divided by the area fraction that the structure occupies per cell area $A$. In the given example one has $A=\lambda_{x} \lambda_{y}$.
The interaction energy of the magnetic structure and the substrate can be evaluated as almost trivial, as it is already expressed in terms of equivalent surface charges. Following Ref. 20 the energy change due to the substrate can be calculated as a surface integral of the form

$$
E^{\text {stat-ind }}=\frac{1}{2} \int \mathrm{d} S \Phi^{\text {stat }} \sigma^{\text {ind }},
$$

which differs by a factor of $1 / 2$ from the simple charge-charge interaction. $^{21}$ To emphasize that the potential is due to the magnetic structure only, the index "stat" is added here.

The energy density reads

$$
\begin{aligned}
\mathcal{E}^{\text {stat-ind }}= & \frac{1}{2 A h} \int_{A} \mathrm{~d} \vec{\rho} \sigma^{\text {ind }} \Phi_{\mathrm{sc}}^{\text {stat }} \\
= & \frac{1}{2 A h} \int_{A} \mathrm{~d} \vec{\rho}\left(\sigma_{0} \sum_{\vec{q}} \mathrm{e}^{2 \pi \mathrm{i} \vec{q} \cdot \vec{\rho}} s_{q}^{\text {ind }}\right) \\
& \times\left(\frac{M_{\mathrm{S}}}{4 \pi} \sum_{\vec{k}} \mathrm{e}^{2 \pi \mathrm{i} \vec{k} \cdot \vec{\rho}} \frac{s_{k}^{\text {stat }}}{|\vec{k}|}\right) .
\end{aligned}
$$

This integral is only nonzero if $\vec{q}=-\vec{k}$ such that the integral becomes independent of $x$ and $y$. The trivial solution reads

$$
\begin{aligned}
\frac{\mathcal{E}^{\text {stat-ind }}}{K_{\mathrm{d}}}= & -\frac{1}{2} \frac{\chi}{2+\chi} \sum_{\vec{q}} \frac{\left(1-\mathrm{e}^{-2 \pi|\vec{q}| h}\right)^{2}}{2 \pi|\vec{q}| h} \\
& \times\left|\mathrm{i} \frac{\vec{a}_{q} \cdot \vec{q}}{|\vec{q}|}+b_{q}\right|^{2} .
\end{aligned}
$$

A Taylor expansion for small $\chi$ reproduces the results of Ref. 12, but without the complicated evaluation of field and induced magnetization and basically without integration except trivial ones.

\section{SPIN-REORIENTATION TRANSITION IN A SQUARE LATTICE OF CUBOIDS}

To emphasize the effect of a strongly polarizable substrate we consider a quadratic grid of cubes. The cubes have $l=$ $w=h$; see Fig. 1 . The grid has a periodicity of $\lambda$ and is on top of a substrate with polarizability $\chi$. Inside the cubes a homogeneous magnetization is assumed, i.e.,

$$
\vec{M}=M_{\mathrm{S}}(\sin \theta \cos \phi, \sin \theta \sin \phi, \cos \theta),
$$

where $\theta$ and $\phi$ are the standard spherical coordinates. In this case the geometric and magnetic part of the Fourier series has the form

$$
\begin{aligned}
\mathrm{i} \frac{\vec{q} \cdot \vec{a}_{q}}{|\vec{q}|}+b_{q}= & \left(\mathrm{i} \frac{\vec{q}\left(\begin{array}{c}
\sin \theta \cos \phi \\
\sin \theta \sin \phi
\end{array}\right)}{|\vec{q}|}+\cos \theta\right) \\
& \times \frac{\sin \pi h q_{x}}{\pi q_{x} \lambda_{x}} \frac{\sin \pi h q_{y}}{\pi q_{y} \lambda_{y}} .
\end{aligned}
$$

The first term contains only the information about the direction of the magnetization while the second term is defined by the particle shape. The self-energy per magnetic volume of the array in the case of magnetization directions in the [100], [001], and [111] directions as a function of the array's periodicity is shown in Fig. 3. Naturally, the energy density per magnetic 

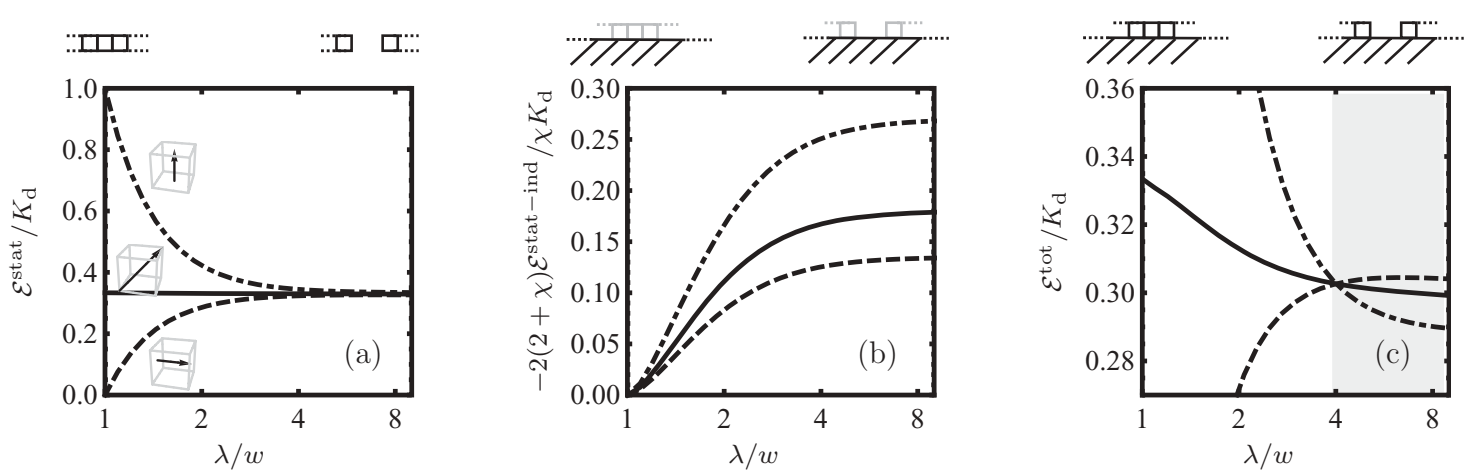

FIG. 3. Energy per magnetic volume of an array of cubes as a function of array periodicity. (a) Self energy of the freestanding array. (b) Corrections due to the substrate. Note that the substrate self-energy shows the same behavior, however, with a different scaling. (c) Total energy $\mathcal{E}^{\text {tot }}$ for $\chi=1$, i.e., a combination of (a) and (b). The curves correspond to magnetization in the [100] (dashed), [001] (doted-dashed), and [111] direction (continuous). Note that the substrate induces a spin-reorientation transition from in-plane, white area in (c), to out-of-plane, gray area in (c).

volume of the freestanding array converges to $K_{\mathrm{d}} / 3$, the demagnetizing energy of a single cube. In case of $\lambda=h$, i.e., the closed film, in-plane magnetization results in zero energy, while out-of-plane magnetization gives the well-known result of $K_{\mathrm{d}}$. Interestingly, in the case of a magnetization in the [111] direction the result is independent of $\lambda$. Hence, the increase in energy due to the in-plane components is exactly compensated by the decrease due to the out-of-plane component. From a mathematical point of view this can be understood looking at Eq. (12). For a magnetization in the [111] direction all $h$-dependent terms in Eq. (12) become odd in $q_{x}$ and $q_{y}$ such they add up to zero in the Fourier sum. Moreover, this property is independent of the particle shape; it only requires that the magnetization is in [111] direction with respect to the array.

In Fig. 3(b) one can see the important impact of the substrate. Although the energy due to the substrate is small compared to the structure's self energy, it introduces a spinreorientation transition (SRT) at finite $\lambda$, which can be seen as a crossing of graphs present in Fig. 3(c) but not present in Fig. 3(a). Note that this is true for all $\chi>0$. For $\lambda=1$ one has a closed film and the substrate plays no role. In all other cases, however, the energy is affected. The energy is lowered most in the case of a $z$ magnetization. Hence, the polarizable substrate shifts the height-dependent SRT for cuboids to smaller $h$. Substrates with $\chi>0$, hence, stabilize a perpendicular magnetization. To show this effect Table I and Fig. 4 compare the SRT of a freestanding array and an array on top of a substrate with $\chi=1$. The critical ratio $w / h$ is set to the point where the total magnetostatic energy is identical for $\vec{M}=(M, 0,0)$ and $\vec{M}=(0,0, M)$. Note that for all $\lambda$ the critical height is reduced by approximately $6 \%$.

TABLE I. Critical ratio $h / w(l=w)$ for the spin-reorientation transition in an array of cuboids on substrates with and without polarizability.

\begin{tabular}{lccccc}
\hline \hline$\lambda$ & 1.8 & 2 & 3 & 5 & $\infty$ \\
\hline$\chi=0$ & 6.26 & 2.16 & 1.15 & 1.03 & 1 \\
$\chi=1$ & 5.92 & 2.04 & 1.08 & 0.97 & 0.95 \\
\hline \hline
\end{tabular}

Pure paramagnetic substrates with $\chi$ close to 1 present a theoretical extreme. Nevertheless, smaller $\chi$ will still have an impact on the SRT and the system stability. The latter is especially the case when referring to the superparamagnetic limit, where small changes in energy affect the system properties exponentially.

\section{SUMMARY}

Formulas to calculate the self-energy of two-dimensional periodic magnetic structures have been presented. The periodicity may be due to a periodic magnetization vector field and/or due to a periodically structured thin film. The effect of a polarizable substrate has been taken into account by introducing equivalent surface charges at the substratemagnetic structure interface. The virtual surface charges allow for an easy calculation of the substrate-magnetic structure interaction. The substrate-magnetic structure interaction can be of importance as, e.g., in patterned media, dot or antidot. In either case the presented formulas allow for an exact calculation of the substrate's influence.

Furthermore, the results may be used as an approximation for SUL. In the case of a soft ferromagnetic material the formulas are a good approximation if bulk charges are

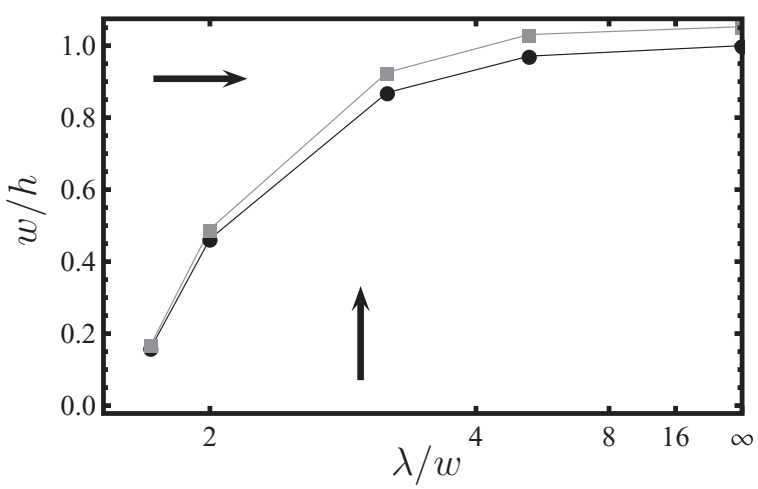

FIG. 4. Phase diagram showing the spin-reorientation transition in a square array of cuboids. The black line corresponds to the critical values of a freestanding array, while the gray curve takes into account a substrate with $\chi=1$. 
negligible ${ }^{22}$ and the surface charge is proportional to the local field. It is, however, worth mentioning that the gain in stability by a drastic change from $\chi=1$ in a paramagnet to $\chi \approx 10^{5}$ in a SUL is far less drastic, as the factor $\chi(2+\chi)^{-1}$ only changes from $1 / 3$ to approximately 1 . The strength of the virtual image moments, hence, only increase by a factor of 3. The importance of a pure paramagnetic substrate has been shown using the example of the spin reorientation in a square array of cuboids.

In summary we have shown that polarizable substrates can significantly influence the energetics of magnetic arrays. Paramagnetic substrates with large susceptibility significantly alter the stray-field energy similar to soft ferromagnetic underlayers, even promoting a spin-reorientation transition. It can be concluded that substrates with tunable polarizability give new possibilities to control the properties of nanodot arrays. $^{23,24}$

\section{ACKNOWLEDGMENTS}

We acknowledge funding by Consolider-Ingenio en Nanociencia Molecular Ref. No. CSD2007-00010, by the Comunidad de Madrid through Project No. S2009/MAT-1726, and Project No. FIS 2010-18847 from MICINN. *nikolai.mikuszeit@imdea.org

${ }^{1}$ F. Meier, Lihui Zhou, J. Wiebe, and R. Wiesendanger, Science 320, 82 (2008).

2 Lihui Zhou, J. Wiebe, L. Samir, E. Vedmedenko, F. Meier, S. Blügel, P. H. Dederichs, and R. Wiesendanger, Nat. Phys. 6, 187 (2010).

${ }^{3}$ A. Moser, Kentaro Takano, D. T. Margulies, M. Albrecht, Yoshiaki Sonobe, Yoshihiro Ikeda, Shouheng Sun, and E. E. Fullerton, J. Phys. D: Appl. Phys. 35, R157 (2002).

${ }^{4}$ M. Mallary, A. Torabi, and M. Benakli, IEEE Trans. Magn. 38, 1719 (2002).

${ }^{5}$ D. Suess, T. Schrefl, S. Fähler, M. Kirschner, H. Hrkac, F. Dorfbauer, and J. Fidler, Appl. Phys. Lett. 87, 012504 (2005).

${ }^{6}$ C. A. Ross, M. Hwang, M. Shima, J. Y. Cheng, M. Farhoud, T. A. Savas, H. I. Smith, W. Schwarzacher, F. M. Ross, M. Redjdal, and F. B. Humphrey, Phys. Rev. B 65, 144417 (2002).

${ }^{7}$ O. D. Kellogg, Foundations of Potential Theory (Dover Publications, Inc., 1953).

${ }^{8}$ J. D. Jackson, Classical Electrodynamics (John Wiley \& Sons, Inc., New York, 1962).

${ }^{9}$ M. Beleggia and M. De Graef, J. Magn. Magn. Mater. 285, L1 (2005).

${ }^{10}$ P. Rhodes and G. Rowlands, Proc. Leeds Phil. Liter. Soc. 6, 191 (1954).

${ }^{11}$ E. Y. Tsymbal, Appl. Phys. Lett. 77, 2740 (2000).

${ }^{12}$ N. Mikuszeit, S. Meckler, R. Wiesendanger, and R. Miranda, Phys. Rev. B 84, 054404 (2011).
${ }^{13}$ J. Kaczér and L. Murtinová, Phys. Status Solidi A 23, 79 (1974).

${ }^{14}$ H. N. Bertram and M. Williams, IEEE Trans. Magn. 36, 4 (2000).

${ }^{15}$ D. Litvinov and S. Khizroev, J. Appl. Phys. 97, 071101 (2005).

${ }^{16}$ B. Lenk, H. Ulrichs, F. Garbs, and M. Münzenberg, Phys. Rep. 507, 107 (2011).

${ }^{17}$ Zhijun Hu, Mingwen Tian, B. Nysten, and A. M. Jonas, Nat. Mater. 8, 62 (2009).

${ }^{18}$ This notation has been chosen to keep the analogy to Mikuszeit et al. (Ref. 12).

${ }^{19}$ M. Beleggia, S. Tandon, Y. Zhu, and M. De Graef, J. Magn. Magn. Mater. 278, 270 (2004).

${ }^{20}$ L. D. Landau and E. M. Lifschitz, Elektrodynamik der Kontinua, Lehrbuch der Theoretischen Physik, Vol. 8 (Akademie-Verlag, Berlin, 1985).

${ }^{21}$ This energy is given by the integral over the force required to bring magnetic structure and substrate together from infinity. As a consequence the energy difference of two states corresponds to the work necessary to make the required transition.

${ }^{22}$ Note that volume charges are unavoidable if the magnitude of the local magnetic moment is not proportional to the field but fixed as in a ferromagnet.

${ }^{23}$ Jungpil Seo, T.-H. Kim, S.-H. Chung, Y. Oh, J. H. Choi, and Young Kuk, Appl. Phys. Lett. 96, 073106 (2010).

${ }^{24}$ A. Ebbing, O. Hellwig, L. Agudo, G. Eggeler, and O. Petracic, Phys. Rev. B 84, 012405 (2011). 